\title{
Scintillation measurements of the millisecond pulsar PSR J0030+0451 and pulsar space velocities
}

\author{
L. Nicastro ${ }^{1}$, F. Nigro ${ }^{2}$, N. D'Amico ${ }^{2}$, V. Lumiella ${ }^{3}$, and S. Johnston ${ }^{4}$ \\ 1 Istituto di Fisica Cosmica con Applicazioni all'Informatica, CNR, Via U. La Malfa 153, 90146 Palermo, Italy \\ 2 Osservatorio Astronomico di Bologna, Via Ranzani 1, 40127 Bologna, Italy \\ 3 Dipartimento di Astronomia, Università degli Studi di Bologna, Via Ranzani 1, 40127 Bologna, Italy \\ 4 Research Centre for Theoretical Astrophysics, University of Sydney, NSW 2006, Australia
}

Received 6 December 2000 / Accepted 12 January 2001

\begin{abstract}
Scintillation observations of the nearby single millisecond pulsar (MSP) PSR J0030+0451 were carried out with the Parkes $64 \mathrm{~m}$ radiotelescope at three different epochs in 1999. From analysis of the dynamic spectrum we obtained the amplitude of the electron density power spectrum $\log \overline{C_{\mathrm{N}}^{2}} \simeq-3.33$ and a scintillation velocity $V_{\text {iss }} \lesssim 15 \mathrm{~km} \mathrm{~s}^{-1}$. This result shows that the Shklovskii effect on the spin-down rate $\dot{P}$ is negligible. We also performed a correlation analysis between pulsar proper motions $\left(V_{\mathrm{pm}}\right)$ and scintillation velocities $\left(V_{\mathrm{iss}}\right)$ using updated measurements for a sample of 77 objects, 17 of which are MSPs. The full sample shows a correlation coefficient $r_{\mathrm{s}} \simeq 80 \%$ at an extremely high significance level, while for the MSP sub-sample (excluding 2 outliers) we obtain $r_{\mathrm{s}} \simeq 90 \%$.
\end{abstract}

Key words. stars: kinematics - pulsars: individual: PSR J0030+0451 - ISM: general - ISM: kinematics and dynamics - ISM: structure - radio continuum: stars

\section{Introduction}

PSR J0030+0451 was independently discovered by the Arecibo Drift Scan Search (Somer 2000) and the Bologna sub-millisecond pulsar survey (D'Amico 2000). It was the first millisecond pulsar (MSP) discovered with the Northern Cross radiotelescope and it appeared to be strongly scintillating at $\lambda=70 \mathrm{~cm}$. Follow-up observations performed at Parkes with the $21 \mathrm{~cm}$ multibeam receiver confirmed the pulse period $P \simeq 4.865 \mathrm{~ms}$, the dispersion measure $(\mathrm{DM}) \simeq 4.33 \mathrm{pc} \mathrm{cm}{ }^{-3}$ and showed the pulsar is not in a binary system. Updated ephemeredes from the ongoing monitoring were used for the data analysis of our scintillation observations.

Given its galactic coordinates $l \simeq 113^{\circ}, b \simeq-57^{\circ}$ and DM, the Taylor \& Cordes (1993) electron distribution model gives a distance $D=0.230 \mathrm{kpc}$. Timing observations of PSR J0030+0451 were carried out by Lommen et al. (2000) using the Arecibo radiotelescope. Among the derived parameters, they report $\dot{P} \simeq(1.0 \pm$ $0.2) 10^{-20} \mathrm{~s} \mathrm{~s}^{-1}$, and an upper limit on the pulsar proper motion of $\mu \lesssim(60 \div 70)$ mas $\mathrm{yr}^{-1}$. The so called "Shklovskii effect" could affect the intrinsic $\dot{P}$ by

$\dot{P}_{\mathrm{s}} / P \simeq 1.110^{-22} V_{\perp}^{2} D_{\mathrm{kpc}}^{-1} \mathrm{~s}^{-1}$,

Send offprint requests to: L. Nicastro, e-mail: nicastro@ifcai.pa.cnr.it where $V_{\perp}$ is the pulsar transverse velocity in $\mathrm{kms}^{-1}$ and $D_{\mathrm{kpc}}$ its distance in kpc (Shklovskii 1970; Camilo et al. 1994). This puts an upper limit on the proper motion of $\mu \lesssim 60 \operatorname{mas} \mathrm{yr}^{-1}$.

MSPs have an observed mean transverse velocity of $85 \pm 13 \mathrm{~km} \mathrm{~s}^{-1}$, a factor $\sim 4$ lower than that of ordinary pulsars, with those in binary systems having about twice the velocity of the isolated ones (Toscano et al. 1999b; Johnston et al. 1998; Nicastro \& Johnston 1995). Improving this statistical sample is particularly important in the light of neutron star birth scenarios. PSR J0030+0451's distance, its peculiar position in the Local Interstellar Medium (LISM) (see Toscano et al. 1999a) and its strong flux variations make this MSP a good target for scintillation studies.

The correlation between pulsar proper motions $\left(V_{\mathrm{pm}}\right)$ and scintillation speeds $\left(V_{\text {iss }}\right)$ was investigated in the past by several authors (e.g. Harrison \& Lyne 1993; Gupta 1995) showing the latter is a good estimate of the pulsar transverse velocity. Here we revisit the analysis on a sample of 77 objects, including PSR J0030+0451 and 16 more MSPs, for which proper motion (13 are high confidence upper limits) and scintillation measuremnts exist. The analysis was performed both using the whole sample and the MSPs subsample only. 


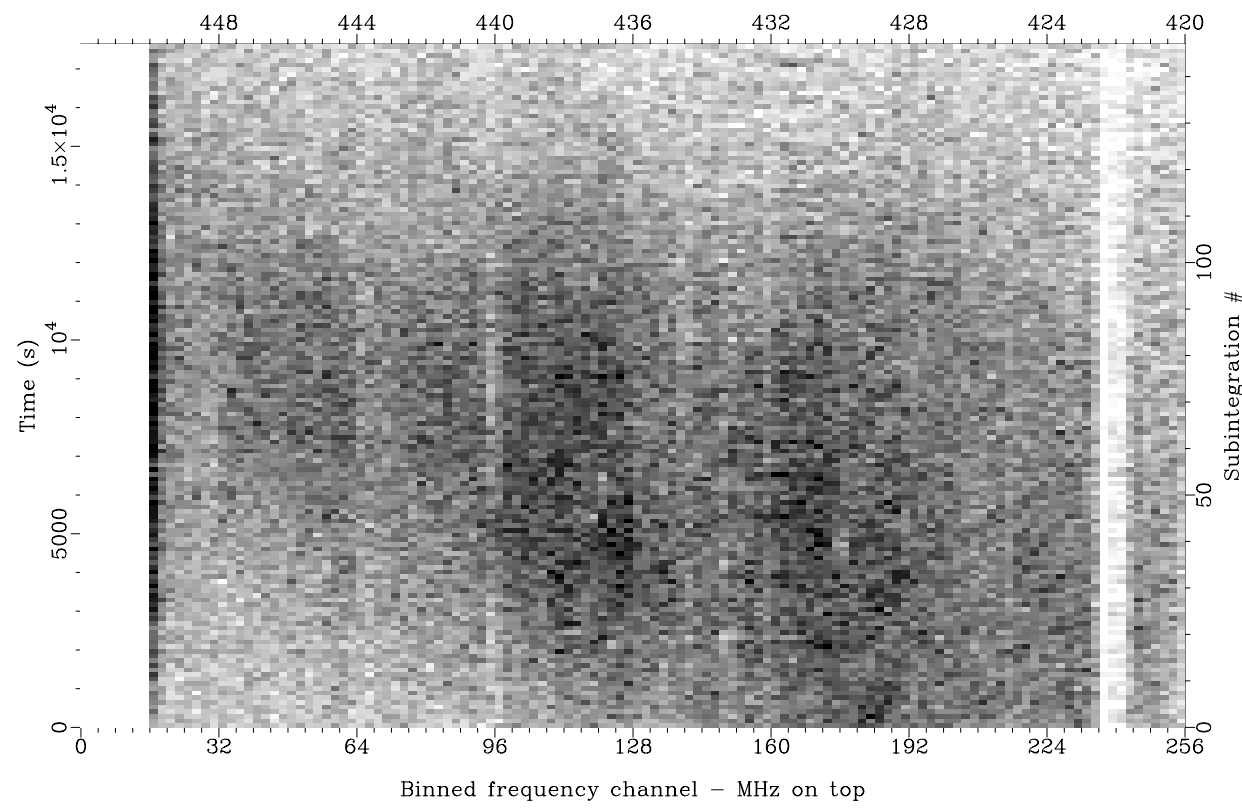

Fig. 1. Dynamic spectrum of PSR J0030+0451 with a frequency resolution of $0.5 \mathrm{MHz}$ and a subintegration time of $960 \mathrm{~s}$. The gray-scale shows the signal strength increasing from white to black ( $\mathrm{S} / \mathrm{N}$ range $2 \div 20$ )

\section{Observations and data analysis}

During 1999, three observations of PSR J0030+0451 were performed with the $64 \mathrm{~m}$ Parkes radiotelescope at a central frequency of $436 \mathrm{MHz}$. The total bandwidth was $32 \mathrm{MHz}$ subdivided into 256 filter channels, each of width $125 \mathrm{kHz}$. The filterbanks are sampled continuously at a fixed rate of $0.05 \mathrm{~ms}$; the output from each channel is one-bit digitized and written to magnetic tape for off-line analysis.

The first observation was performed on 1999 July 24 and lasted $7000 \mathrm{~s}$. The integrated pulsar signal was relatively strong, but unfortunately the observation time was short compared to the diffractive scintillation time and only part of the scintles are visible in the frequency-time dynamic spectrum. Since this MSP has a northern declination, the longest observation time from Parkes is about 5.5 hours, which was the duration of two more observations performed on October 15 and 16. In both cases the pulsar signal was weaker than in the first observation though we were able to detect a number of scintles in the dynamic spectra.

During the analysis, pulse profiles are formed at the apparent pulsar period over short sections in time and for each of the 256 filter channels. Subintegration times were $60 \mathrm{~s}$ for the first observation and $120 \mathrm{~s}$ for the other two. The signal-to-noise ratios in each subintegration for each frequency channel is then calculated. The first 16 channels were not considered in the analysis because of their malfunctioning; a few other channels that had bad response or in which strong interferences were present, were overwritten by an average of the signal-to-noise ratios in adjacent channels. No smoothing operation was made on the dynamic spectra but in all the observations a sum along the frequency and time axes was performed in order to im- prove the signal-to-noise ratio. Figure 1 shows a dynamic spectrum of PSR J0030+0451.

To obtain the scintillation bandwidth and decorrelation time-scale we performed a two-dimensional autocorrelation analysis on the dynamical spectra, following the method described by Cordes (1986). The scintillation bandwidth, $\Delta \nu_{\mathrm{d}}$, and the decorrelation time-scale, $t_{\mathrm{d}}$, were obtained by fitting a Gaussian function to the zero lag in frequency and time respectively (details on the data analysis and description of the software used can be found in Nigro 2000). Similar results were obtained with a 2-dimensional Gaussian fit.

\section{Results and discussion}

Scintillation velocities $\left(V_{\text {iss }}\right)$ can be estimated using the equation (Gupta et al. 1994)

$V_{\text {iss }}=3.8510^{4} \frac{\sqrt{\Delta \nu_{\mathrm{d}} D_{\mathrm{kpc}} x}}{\nu_{\mathrm{GHz}} t_{\mathrm{d}}} \mathrm{km} \mathrm{s}^{-1}$,

while the line-of-sight-averaged electron density fluctuations can be estimated by (Cordes et al. 1985; Cordes 1986)

$\overline{C_{\mathrm{N}}^{2}}=0.002 \nu_{\mathrm{GHz}}^{11 / 3} D_{\mathrm{kpc}}^{-11 / 6} \Delta \nu_{\mathrm{d}}^{-5 / 6} \mathrm{~m}^{-20 / 3}$,

where $C_{\mathrm{N}}^{2}$ is the amplitude of the (density fluctuations) power spectrum, $\Delta \nu_{\mathrm{d}}$ is the decorrelation bandwidth in $\mathrm{MHz}, t_{\mathrm{d}}$ the characteristic time-scale in $\mathrm{s}, D_{\mathrm{kpc}}$ the distance in $\mathrm{kpc}, \nu_{\mathrm{GHz}}$ the observation frequency in $\mathrm{GHz}$ and $x \equiv D_{\mathrm{o}} / D_{\mathrm{p}}$ is the scaling factor that accounts for an asymmetrically located scattering screen, whith $D_{\mathrm{o}}$ and $D_{\mathrm{p}}$ the distances observer-screen and screen-pulsar, respectively. Apart (identifiable) peculiar cases, assuming $x=1$ and a thin screen model (Scheuer 1968) gives good agreements between $V_{\mathrm{pm}}$ and $V_{\text {iss }}$ (see below). 
Table 1. PSR J0030+0451 observation log and scintillation parameters

\begin{tabular}{ccccccc}
\hline Date & $T$ & $V_{\oplus}$ & $\begin{array}{c}\Delta \nu_{\mathrm{d}} \\
(\text { hours })\end{array}$ & $\begin{array}{c}t_{\mathrm{d}} \\
\left(\mathrm{km} \mathrm{s}^{-1}\right)\end{array}$ & $\begin{array}{c}V_{\mathrm{iss}}^{*} \\
(\mathrm{MHz})\end{array}$ & $\begin{array}{c}\log \overline{C_{N}^{2}} \\
(\mathrm{~s})\end{array}$ \\
\hline $24-07-1999$ & 1.9 & 11.7 & 9.3 & 10569 & $12.5 \pm 11.9$ & -3.66 \\
$15-10-1999$ & 5.5 & 29.2 & 4.9 & 10106 & $9.4 \pm 4.0$ & -3.43 \\
$16-10-1999$ & 5.5 & 29.1 & 2.0 & 9518 & $6.4 \pm 1.6$ & -3.10 \\
\hline
\end{tabular}

* Errors are $1 \sigma$ level from Eq. (4).

Table 1 lists the scintillation parameters of the three observations. Columns 2 to 5 give observation time, transverse Earth velocity toward the pulsar, decorrelation bandwidth, characteristic time-scale, scintillation velocity and the measure of the turbulence along the line of sight. The statistical error on $V_{\text {iss }}$ is a combination of two quantities: the uncertainties on $\Delta \nu_{\mathrm{d}}$ and $t_{\mathrm{d}}$ from the Gaussian fitting, and the empirical error of the scintillation measurements given by Bhat et al. (1998) as

$\sigma=\left[f_{\mathrm{d}}\left(\frac{B_{\mathrm{obs}} t_{\mathrm{obs}}}{\Delta \nu_{\mathrm{d}} t_{\mathrm{d}}}\right)\right]^{-0.5}$

where $\sigma$ is the fractional error, $B_{\mathrm{obs}}$ and $t_{\mathrm{obs}}$ are the observation bandwidth and time respectively, and $f_{\mathrm{d}}$ is the filling fraction for number of scintles which here is assumed to be 0.5 . This estimate uncertainty is far larger than that from the fit and that due to the dynamic spectra discretization, so these two latter are assumed to be negligible.

The scintillation velocity is a combination of (projected on the plane of the sky perpendicular to the direction of the pulsar) the pulsar velocity $V_{\mathrm{pm}}$, ISM irregularities velocity $\sigma_{V_{\text {ISM }}}$ (assumed to be $\sim 10 \mathrm{~km} \mathrm{~s}^{-1}$ ) and Earth orbital velocity $V_{\oplus}$ (varying from 0 to $\sim 30 \mathrm{~km} \mathrm{~s}^{-1}$ ). In the case of a binary system, the pulsar's orbital velocity should also be considered. For pulsars with high values of $V_{\text {iss }}, \sigma_{V_{\text {ISM }}}$ and $V_{\oplus}$ give a negligible contribution to the estimate of the transverse velocity (see e.g. Gupta et al. 1994). For PSR J0030+0451 the values of $V_{\text {iss }}$ are similar to $\sigma_{V_{\mathrm{ISM}}}$ and in two, out of three cases, $V_{\text {iss }}<V_{\oplus}$. Therefore these velocities contribute significantly to the uncertainty. A possible way to consider these systematics is by

$\sigma_{V_{\perp}}=\sigma_{V_{\mathrm{iss}}}+\frac{\sqrt{\sigma_{V_{\mathrm{ISM}}^{2}}^{2}+V_{\oplus}^{2}}}{2}$

However, to better estimate the velocity $V_{\perp}$ of the pulsar and the related uncertainty, a more reliable way would be by performing a campaign of measurements preferably when the contribution of $V_{\oplus}$ is minimum $\left(\sim 5 \mathrm{~km} \mathrm{~s}^{-1}\right)$. In addition, having several measurements would allow us to assume $\overline{V_{\text {ISM }}}=0$. Neverthless our measurements clearly indicate PSR J0030+0451 is a slowly moving object.

\subsection{LISM}

The average amplitude of the electron density power spectrum value $\log \overline{C_{\mathrm{N}}^{2}}=-3.33$ we found for PSR J0030+0451 is somewhat greater than -3.5 , the average value for the whole pulsar sample with distances $\gtrsim 1 \mathrm{kpc}$, i.e. out of the LISM (Cordes 1986). This estimate adds to that of the other $\sim 50$ pulsars with DM $\lesssim 35 \mathrm{pc} \mathrm{cm}^{-3}$ (20 by Bhat et al. 1998, and 31 by Johnston et al. 1998). Most of these pulsars are localized within the LISM and have high $\overline{C_{\mathrm{N}}^{2}}$ values, confirming the existence of local enhanced scattering. The overall picture shows clearly that the LISM is highly turbulent compared to the outer regions. Bhat et al. (1998) developed a model which they claim can fit the $\overline{C_{\mathrm{N}}^{2}}$ observed in their pulsar sample. It is interesting to check their model against PSR J0030+0451 results since it is located in the quadrant where no other MSPs are known (Toscano et al. 1999a).

The expected decorrelation bandwidth value, given by

$\Delta \nu_{\mathrm{d}, \mathrm{t}} \simeq 11 \nu_{\mathrm{GHz}}^{22 / 5} D_{\mathrm{kpc}}^{-11 / 5}=7.2 \mathrm{MHz}$,

is not too different from the mean measured value $\Delta \nu_{\mathrm{d}}=$ 5.6 MHz. We computed the so called "anomaly parameter" (defined as $A_{\mathrm{dm}}=\left(\nu_{\mathrm{d}_{1}} / \nu_{\mathrm{d}_{2}}\right)_{\mathrm{obs}} /\left(\nu_{\mathrm{d}_{1}} / \nu_{\mathrm{d}_{2}}\right)_{\exp }$, see Bhat et al. 1998) for PSR J0030+0451 with respect to PSR J1133+16 and PSR J1929+10, from the Bhat et al. (1998) sample, and PSR J1744-1134 and J2124-3358 (both MSPs) from the Johnston et al. (1998) sample, as they all have similar distances and, more importantly, DMs within $\simeq 1 \mathrm{pc} \mathrm{cm}^{-3}$. The low values of $A_{\mathrm{dm}}$ we obtain (1.4, 2.4, 1.60 and 1.34 respectively) do not confirm the decay trend shown in Fig. 7 of Bhat et al. (1998). An asymmetry in the distribution of electrons in the LISM (as suggested by Toscano et al. 1999a) or a non-Kolmogorov density spectrum could explain the discrepancy. This is also shown in Fig. 2 where we plot $A_{\mathrm{dm}}$ vs. DM for 35 PSRs (16 MSPs) with $\mathrm{DM}<35 \mathrm{pc} \mathrm{cm}^{-3}$. This sample includes 32 objects from Johnston et al. (1998) and the 3 MSPs: PSR J0030+0451, J1012+5307 and B1257+12. The plot was made by averaging the $A_{\mathrm{dm}}$ of pulsars with DM differences less than $3 \mathrm{pc} \mathrm{cm}^{-3}$ and increasing the DM by that of the pulsars instead of a fixed step of $1 \mathrm{pc} \mathrm{cm}^{-3}$ (as is done by Bhat et al. 1998). Error bars are inversely proportional to the number of pulsar products averaged. The three very high peaks at DMs 10, 21 and $32 \mathrm{pc} \mathrm{cm}^{-3}$ are due to the large discrepancies between the expected decorrelation bandwidths and the measured ones for PSRs J1730-2304, J1825-0935 and J2129-5718, respectively. The estimators Bhat et al. (1998) use to define the best model for the local scattering structure are in our case $\epsilon_{\mathrm{A}}(\mathrm{DM})=0.119$ and $\epsilon_{\mathrm{b}}=0.275$, confirming the inhomogeneity of the LISM.

However the high variability of the observed decorrelation bandwidth in PSR J0030+0451 by a factor $\sim 5$ is rather unusual. This is not caused by refractive scintillation because the dynamic spectra show no frequency drift. Instead it likely originates from anisotropies in the density or the magnetic field structure of the LISM. Further and 


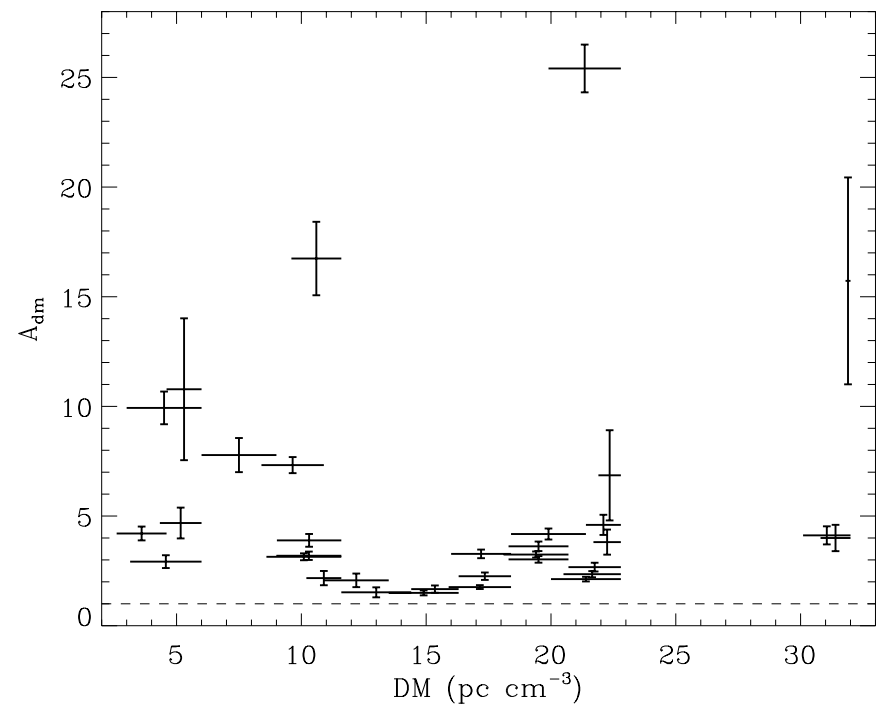

Fig. 2. "Anomaly factor" vs. DM for 35 PSRs with DM < $35 \mathrm{pc} \mathrm{cm}^{-3}$. The 3 isolated highest points are due to PSRs J1730-2304, J1825-0935 and J2129-5718

more sensitive observations are required to put any strong constraint on the proposed model for the LISM by Bhat et al. (1998).

\subsection{Transverse velocity}

The scintillation parameters allow us to derive a reliable estimate of the transverse velocity of PSR J0030+0451. We will show that the correlation between $V_{\text {iss }}$ and $V_{\mathrm{pm}}$ is very high and a relation $V_{\text {iss }} \equiv V_{\text {pm }}$ can in general be assumed (see also Gupta 1995). As already mentioned, in spite of an average high flux density measured during the first observation, because of its short duration compared to $t_{\mathrm{d}}$, the $V_{\text {iss }}$ estimate is affected by a relatively large error. Further, the data of the second and third observation have lower statistical significance, so they can provide only upper limits (see also Table 1). This suggests giving similar weights to the three observations in the calculation of $\overline{V_{\text {iss }}}$. Hence we have determined a mean velocity of $\overline{V_{\perp}} \simeq 9 \pm 6 \mathrm{kms}^{-1}$ ( $1 \sigma$ error $)$. This number makes PSR J0030+0451 the pulsar with the smallest transverse velocity so far measured. Its position in the $V_{\mathrm{pm}}$ vs. $V_{\text {iss }}$ scatter plot is quite isolated in the bottom left corner, well in agreement with a correlation $V_{\mathrm{pm}} \equiv V_{\text {iss }}$ (see Fig. 3 ).

If we assume that the $\dot{P}=(1 \pm 0.2) 10^{-20}$ reported by Lommen et al. (2000) is all due to the Shklovskii effect, we obtain an upper limit to the transverse speed $V_{\perp}<$ $65 \mathrm{~km} \mathrm{~s}^{-1}$. Now assuming $\overline{V_{\perp}}=9 \mathrm{~km} \mathrm{~s}^{-1}$, the derived contribution to the spin-down rate is $\dot{P}_{\mathrm{s}}<210^{-22} \mathrm{~s} \mathrm{~s}^{-1}$. This means that the Shklovskii effect introduces an error of only $\sim 2 \%$ on $\dot{P}=\dot{P}_{i}+\dot{P}_{\mathrm{s}}$, lower than the $\sim 20 \%$ so far obtained by the timing data fit. Hence we can assume the measured spin-down rate is the intrinsic one: $\dot{P}_{i}=$ $(1.0 \pm 0.2) 10^{-20} \mathrm{~s} \mathrm{~s}^{-1}$ (Lommen et al. 2000). However, using a $3 \sigma$ upper limit $\overline{V_{\perp}} \simeq 27 \mathrm{~km} \mathrm{~s}^{-1}$, we obtain a $\dot{P}_{\mathrm{s}}$ comparable to the $\dot{P}$ error estimate. We then conclude that $\dot{P}_{i} \cong(0.8 \div 1) 10^{-20} \mathrm{~s} \mathrm{~s}^{-1}$, giving a surface magnetic field $B \cong(2 \div 2.2) 10^{8} \mathrm{G}$ and a characteristic age $\tau \cong$ $(8 \div 10) 10^{9}$ years.

This pulsar was also detected in the soft X-ray band (Becker et al. 2000). Its X-ray luminosity $(0.1-2.4 \mathrm{keV})$ is $L_{x} \sim(1 \div 2) 10^{30}(d / 0.23 \mathrm{kpc})^{2} \mathrm{erg} \mathrm{s}^{-1}$. Using the inferred rotational energy loss rate $\dot{E}=(2.7 \div 3.4) 10^{33} \mathrm{erg} \mathrm{s}^{-1}$ we obtain a relatively narrow $\mathrm{X}$-ray efficiency range $\eta=$ $L_{x} / \dot{E} \simeq(0.3 \div 0.7) 10^{-3}(d / 0.23 \mathrm{kpc})^{2}$. This is in good agreement with the proposed law $L_{x} / \dot{E} \simeq 10^{-3}$ (Becker \& Trümper 1997) and points toward a magnetospheric origin for the high energy radiation, in spite the inferred rotational energy loss rate and pulse period would put PSR J0030+0451 in the class of MSPs for which the X-ray emission is believed to be mostly thermal (Kawai \& Saito 1999). Becker et al. (2000), from the observed X-ray pulse profile (very similar to the radio one) and the large pulsed fraction ( $\sim 69 \pm 18 \%$ ), also suggest a non-thermal origin of the X-ray emission. Thanks to the Chandra and XMMNewton X-ray satellites, a good quality X-ray spectrum should soon be able to give a definitive answer in this respect.

PSR J0030+0451 is the 8th isolated MSP detected for which a transverse velocity is available. Using for PSR $\mathrm{J} 0030+0451 V_{\mathrm{pm}}=32 \mathrm{~km} \mathrm{~s}^{-1}$ (i.e. $\left.\simeq 65 / 2\right)$, the $\overline{V_{\mathrm{pm}}}$ for 7 MSPs (we exclude PSR J1730-2304) is $58 \pm 6 \mathrm{~km} \mathrm{~s}^{-1}$ (for $5 \operatorname{MSPs} \overline{V_{\text {iss }}} \simeq 45 \mathrm{~km} \mathrm{~s}^{-1}$ ). This, even if at low statistical significance, is nearly a factor 2 lower than the $96 \pm 9 \mathrm{~km} \mathrm{~s}^{-1}$ found for 18 binary MSPs (for 12 MSPs $\overline{V_{\text {iss }}} \simeq 113 \mathrm{~km} \mathrm{~s}^{-1}$ ) (Johnston et al. 1998; Toscano et al. 1999b).

\section{4. $V_{\mathrm{pm}}-V_{\text {iss }}$ correlation}

Gupta (1995) made a comparison between proper motion and scintillation velocity for 59 pulsars using the Taylor \& Cordes (1993) model to calculate their distances and a new formula to calculate the ISS velocities (Gupta et al. 1994). The comparison showed a good correlation between $V_{\mathrm{pm}}$ and $V_{\text {iss }}$. The correlation coefficient he found is $r=0.42$. It becomes 0.47 if pulsars for which only upper limits were available are excluded from the sample. A least square fit gave a slope of $1.1 \pm 0.13$.

We updated the $V_{\mathrm{pm}}-V_{\text {iss }}$ diagram

1. adding the proper motion and scintillation speeds of 16 MSPs (Toscano et al. 1999b; Lange et al. 2000; Johnston et al. 1998; Gothoskar \& Gupta 2000; Lorimer et al. 1995; this paper) and of the relativistic pulsar B1534+12 (Stairs et al. 1998);

2. using 14 revised proper motion estimates (Fomalont et al. 1997; Fomalont et al. 1999);

3. using revised values of $V_{\text {iss }}$ for 22 pulsars (Bhat et al. 1999; Lumiella 2000), plus PSR B1259-63 (McClureGriffiths et al. 1998).

The $V_{\mathrm{pm}}$ vs. $V_{\text {iss }}$ values for 77 pulsars are shown in the scatter plot of Fig. 3 and listed in Table 2, (MSPs are listed at the bottom of the table). The uncertainties on 
Table 2. Proper motion and scintillation velocities for 77 pulsars

\begin{tabular}{|c|c|c|c|c|c|c|c|c|c|}
\hline $\begin{array}{c}\text { PSR } \\
(\mathrm{J} 2000)\end{array}$ & $\begin{array}{c}\overline{V_{\mathrm{pm}}} \\
\left(\mathrm{km} \mathrm{s}^{-1}\right)\end{array}$ & $\begin{array}{c}\delta \overline{V_{\mathrm{pm}}} \\
(\%)\end{array}$ & $\begin{array}{c}\overline{V_{\mathrm{iss}}} \\
\left(\mathrm{km} \mathrm{s}^{-1}\right)\end{array}$ & $\begin{array}{c}\delta \overline{V_{\text {iss }}} \\
(\%)\end{array}$ & $\begin{array}{c}\text { PSR } \\
(\mathrm{J} 2000)\end{array}$ & $\begin{array}{c}\overline{V_{\mathrm{pm}}} \\
\left(\mathrm{km} \mathrm{s}^{-1}\right)\end{array}$ & $\begin{array}{c}\delta \overline{V_{\mathrm{pm}}} \\
(\%)\end{array}$ & $\begin{array}{c}\overline{V_{\mathrm{iss}}} \\
\left(\mathrm{km} \mathrm{s}^{-1}\right)\end{array}$ & $\begin{array}{c}\delta \overline{V_{\text {iss }}} \\
(\%)\end{array}$ \\
\hline $0304+1932$ & 167 & 21 & 189 & 15 & $1907+4002$ & 126 & 27 & 1179 & 11 \\
\hline $0323+3944$ & 237 & 23 & 163 & 14 & $1913-0440$ & $<372$ & & 380 & 14 \\
\hline $0332+5434$ & 145 & 7 & $186^{*}$ & 9 & $1921+2153$ & $130^{*}$ & 27 & $173^{*}$ & 9 \\
\hline $0358+5413$ & $135^{*}$ & 26 & 488 & 18 & $1932+1059$ & $71^{*}$ & 8 & $159^{*}$ & 12 \\
\hline $0452-1759$ & $320 *$ & 62 & $96^{*}$ & 20 & $1935+1616$ & $480^{*}$ & 21 & 418 & 13 \\
\hline $0454+5543$ & 202 & 12 & 404 & 21 & $1939+2134$ & $<146$ & & 205 & 25 \\
\hline $0528+2200$ & 228 & 99 & 375 & 22 & $1946+1805$ & 37 & 71 & $61^{*}$ & 12 \\
\hline $0543+2329$ & 376 & 47 & 574 & 14 & $1954+2923$ & 87 & 45 & 63 & 27 \\
\hline $0614+2229$ & $<304$ & & 37 & 20 & $1955+5059$ & 495 & 12 & 1175 & 12 \\
\hline $0629+2415$ & $<538$ & & 78 & 48 & $2018+2839$ & $<31$ & & $56^{*}$ & 16 \\
\hline $0630-2834$ & $390^{*}$ & 38 & $170^{*}$ & 8 & $2022+2854$ & 97 & 23 & $250^{*}$ & 7 \\
\hline $0659+1414$ & $251^{*}$ & 19 & 176 & 28 & $2022+5154$ & $91^{*}$ & 2 & 239 & 20 \\
\hline $0814+7429$ & 75 & 18 & 89 & 32 & $2046-0421$ & $<532$ & & 328 & 12 \\
\hline $0820-1350$ & 376 & 13 & 295 & 16 & $2046+1540$ & 159 & 54 & 202 & 12 \\
\hline $0823+0159$ & $<127$ & & 53 & 23 & $2048-1616$ & $355^{*}$ & 4 & $501^{*}$ & 5 \\
\hline $0826+2637$ & 196 & 3.3 & $279^{*}$ & 22 & $2113+2754$ & 381 & 6 & 1105 & 24 \\
\hline $0835-4510$ & 141 & 4 & $136^{*}$ & 20 & $2116+1414$ & $<618$ & & 171 & 26 \\
\hline $0837+0610$ & 174 & 11 & $214^{*}$ & 16 & $2157+4017$ & 485 & 8 & 1316 & 8 \\
\hline 0908-1739 & 142 & 32 & 394 & 31 & $2219+4754$ & 375 & 31 & 402 & 22 \\
\hline $0922+0638$ & 919 & 72 & $642^{*}$ & 4 & $2225+6535$ & 1686 & 2.3 & 6772 & 18 \\
\hline $0953+0755$ & 21 & 28 & $33^{*}$ & 20 & $2305+3100$ & 661 & 28 & 140 & 25 \\
\hline $1115+5030$ & 142 & 8 & 235 & 18 & $0030+0451 \dagger$ & 32 & 100 & 9 & 66 \\
\hline $1136+1551$ & 475 & 1.6 & $396^{*}$ & 40 & $0437-4715$ & 121 & 0.2 & 170 & 15 \\
\hline $1239+2453$ & 303 & 4.4 & $271^{*}$ & 17 & $0711-6830 \dagger$ & 78 & 2.5 & 81 & 20 \\
\hline $1456-6843$ & 89 & 3.4 & $41^{*}$ & 8 & $1012+5307$ & $62^{1}$ & 0.5 & 50 & 40 \\
\hline $1509+5531$ & 913 & 5 & $451^{*}$ & 8 & $\mathrm{~B} 1257+12$ & 284 & 1 & $225^{2}$ & 20 \\
\hline $1543-0620$ & $<170^{*}$ & & $80^{*}$ & 8 & $1455-3330$ & 100 & 54 & 81 & 18 \\
\hline $1543+0929$ & 144 & 40 & 122 & 13 & B1534+12 & 132 & 8 & 161 & 20 \\
\hline $1607-0032$ & $<66$ & & $60^{*}$ & 8 & $1603-7202$ & 27 & 7 & 81 & 28 \\
\hline $1645-0317$ & 660 & 42 & 562 & 16 & $1713+0747$ & $35^{1}$ & 3.2 & 29 & 21 \\
\hline $1709-1640$ & $<70^{*}$ & & 132 & 19 & $1730-2304 \dagger$ & 79 & 35 & 62 & 18 \\
\hline $1752-2806$ & $45^{*}$ & 88 & 55 & 20 & $1744-1134 \dagger$ & $36^{1}$ & 1.8 & 31 & 19 \\
\hline $1807-0847$ & $85^{*}$ & 82 & $95^{*}$ & 11 & $1911-1114$ & 183 & 54 & 180 & 23 \\
\hline $1820-0427$ & 270 & 13 & 250 & 10 & $2051-0827$ & 14 & 57 & 100 & 22 \\
\hline $1823+0550$ & $<238$ & & 62 & 32 & $2124-3358 \dagger$ & 53 & 3.8 & 40 & 23 \\
\hline $1825-0935$ & $75^{*}$ & 60 & $79^{*}$ & 43 & $2129-5718$ & 56 & 50 & 130 & 26 \\
\hline $1840+5640$ & 286 & 12 & 529 & 12 & $2145-0750$ & $58^{1}$ & 11 & 51 & 17 \\
\hline $1844+1454$ & 481 & 25 & 424 & 11 & $2317+1439$ & 80 & 16 & 100 & 20 \\
\hline
\end{tabular}

* Updated velocity estimates respect to Gupta (1995). † Isolated MSP.

${ }^{1}$ Lange et al. (2000), ${ }^{2}$ Gothoskar \& Gupta (2000).

$V_{\text {iss }}$ were computed according to Bhat el al. (1998) except for PSR B1257+12 and B1534+12 for which we gave a $20 \%$ error. For the uncertainties on $V_{\mathrm{pm}}$ we took into account the proper motion errors only. We know that uncertainties in the distance from the Taylor \& Cordes model are on average $\sim 30 \%$ but we do not include this source of error. Only in a few cases do distances come from parallax measurements.

For PSR J0030+0451 we adopted a (low statistical significance) proper motion value of $V_{\mathrm{pm}}=32 \pm$ $32 \mathrm{~km} \mathrm{~s}^{-1}$ (from the Shklovskii upper limit) and $V_{\text {iss }}=$ $9 \pm 6 \mathrm{~km} \mathrm{~s}^{-1}$ (that is the average of the values of Table 1). 


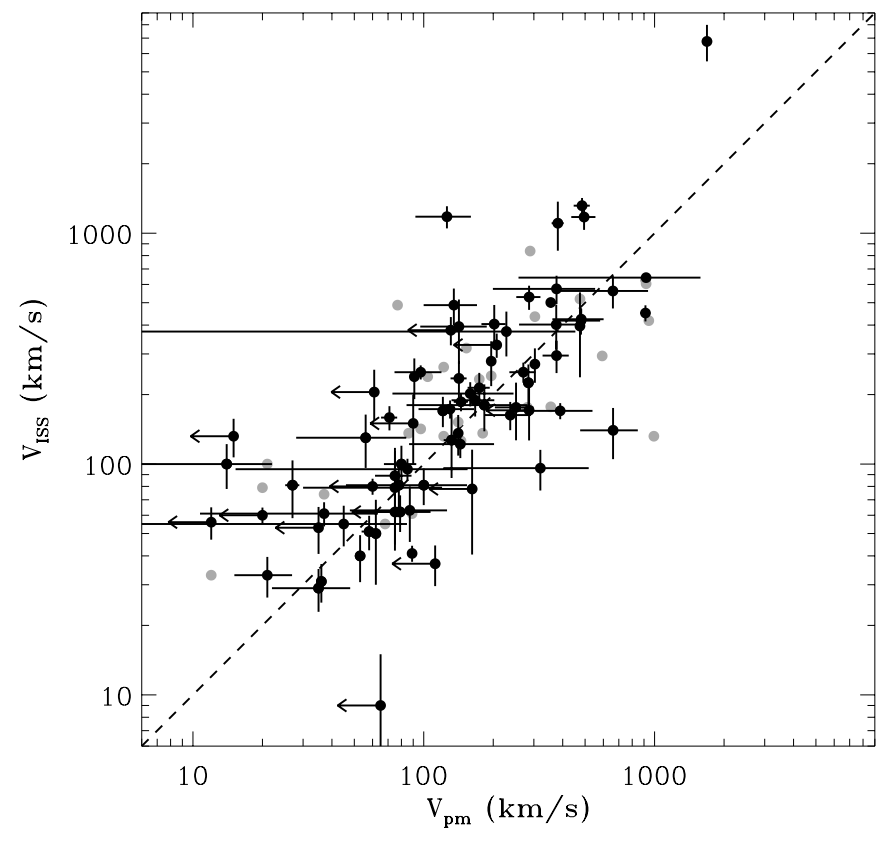

Fig. 3. Scatter diagram for proper motion and scintillation speeds for 77 pulsars. The grey points are the pulsars with updated values compared to Gupta (1995). The $V_{\mathrm{pm}} \equiv V_{\text {iss }}$ correlation line is shown

The object at the top of the plot is PSR J2225+6535. Toscano et al. (1999a) gave for PSR J1730-2304 a lower limit of $V_{\mathrm{pm}} \gtrsim 51 \mathrm{~km} \mathrm{~s}^{-1}$ and an upper limit of $V_{\mathrm{pm}} \lesssim$ $107 \mathrm{~km} \mathrm{~s}^{-1}$ from the Shklovskii effect. We averaged these value and used the estimate of $79 \pm 28 \mathrm{~km} \mathrm{~s}^{-1}$. For the relativistic pulsar PSR B1534+12 we used the inferred distance $D=1.1 \mathrm{kpc}$ obtained by Stairs et al. (1998) assuming the validity of general relativity theory.

A correlation analysis gives for the total sample a coefficient $r=75 \%$, much higher than the value obtained by Gupta (1995). Further statistical analysis, using a rank Spearman test, gives a correlation coefficient $r_{\mathrm{s}}=79 \%$ at an extremely significant level of $\sim 10^{-17}$ (the lower the value the higher the confidence of the result, Press et al. 1992). A least squares fit with a linear law gives a slope of $0.81 \pm 0.21$. Changing the axes of the scatter plot (therefore taking into account the $V_{\mathrm{pm}}$ error values) we obtain a slope of $0.71 \pm 0.20$. Excluding the 14 points with upper limits on $V_{\mathrm{pm}}$, we obtain $r=78 \%$ and $r_{\mathrm{s}}=80 \%$ at a significance level of $\sim 10^{-15}$ and a slope of $0.88 \pm 0.24$. Exchanging the axes of the plot, the least squares fit gives an angular coefficient of $0.70 \pm 0.25$. So we may estimate the slope of a straight line that fits the points taking into account the errors in both axes as $m \simeq 0.90 \pm 0.21$ for the whole sample, and $m \simeq 0.82 \pm 0.25$ excluding the upper limits.

\subsection{MSP correlation}

PSRs J1603-7202 and J2051-0827 are the MSPs for which $V_{\text {iss }}$ differs significantly from $V_{\mathrm{pm}}$. To have $V_{\mathrm{pm}} \equiv$ $V_{\text {iss }}$ the scaling factor should be $x \simeq 3.0$ and $x \simeq 7.1$, re- spectively. These values suggest the presence of a scattering medium near the neutron star. This result is in agreement with the theory that the binary system containing PSR J1603-7202 evolved through a phase with critical unstable mass-transfer and most likely hosts a CO-WD companion rather than a He-WD companion (Tauris \& Savonije 1999). Binary systems like this are believed to be surrounded by an envelope that slowly expands giving rise to the mass transfer (van den Heuvel 1994). This envelope is thought to be the main cause of scintillation. For the eclipsing binary pulsar PSR J2051-0827, the scintillation parameters vary significantly with orbital phase (Stappers et al. 1996; Johnston et al. 1998). It is likely that the wind blown from the companion star is contributing to the scintillation parameters. In these cases, because the screen is so close to the pulsar, the measured scintillation velocity is effectively the velocity of the screen (see e.g. Eq. (3) in Britton et al. 1998). In both cases, the wind could easily have a velocity of $\sim 100 \mathrm{kms}^{-1}$. One possible test of this idea would be to perform VLBI observations of these two pulsars to resolve (or not) the scattering disk; such an observation would give a direct measurement of the screen distance.

We performed a correlation analysis between $V_{\mathrm{pm}}$ and $V_{\text {iss }}$ for the sub-sample of 16 MSPs plus PSR B1534+12. Figure 4 shows the scatter plot. A simple correlation analysis gives a coefficient $r=60 \%$. A rank Spearman test gives a correlation coefficient $r_{\mathrm{s}}=67 \%$ at a significance level of $\sim 310^{-3}$. A least squares fit with a linear law gives a slope of $\sim 0.57$. Exchanging the axis of the scatter plot the least squares fit gives a slope of $\sim 0.47$. Excluding the two above mentioned MSPs from the analysis, the correlation coefficient becomes $r=87 \%$ and $r_{\mathrm{s}}=91 \%$ at a much higher significance level of $\sim 310^{-6}$. A least squares fit with a linear law gives a slope of $\sim 1.1$. Exchanging the axis of the plot the slope becomes $\sim 0.5$. So we estimate the slope of the linear fit to be $m \sim 1.1$ for the whole sample, and $m \sim 1.6$ excluding PSR J1603-7202 and J2051-0827.

Since MSPs have low speeds, a rigorous analysis should take properly into consideration systematical uncertainties due to the Earth orbital velocity and the ISM irregular motion. In spite of that, the resulting $V_{\mathrm{pm}}-V_{\mathrm{iss}}$ correlation is excellent. This demonstrates that averaging several observations spread over an Earth orbit is (in general) sufficient to "wash out" systematics.

\section{Conclusions}

We presented observational and derived parameters for the diffractive scintillation of the recently discovered PSR J0030+0451. Our results for this source show that:

- its decorrelation bandwidth is highly variable;

- the Kolmogorov coefficient $\log \overline{C_{\mathrm{N}}^{2}}=-3.33$ along its line of sight is somewhat higher than the average for pulsars out of the LISM where it is $\log \overline{C_{\mathrm{N}}^{2}}=-3.5$. The enhanced turbulence and strong changes in $\nu_{\mathrm{d}}$ support 


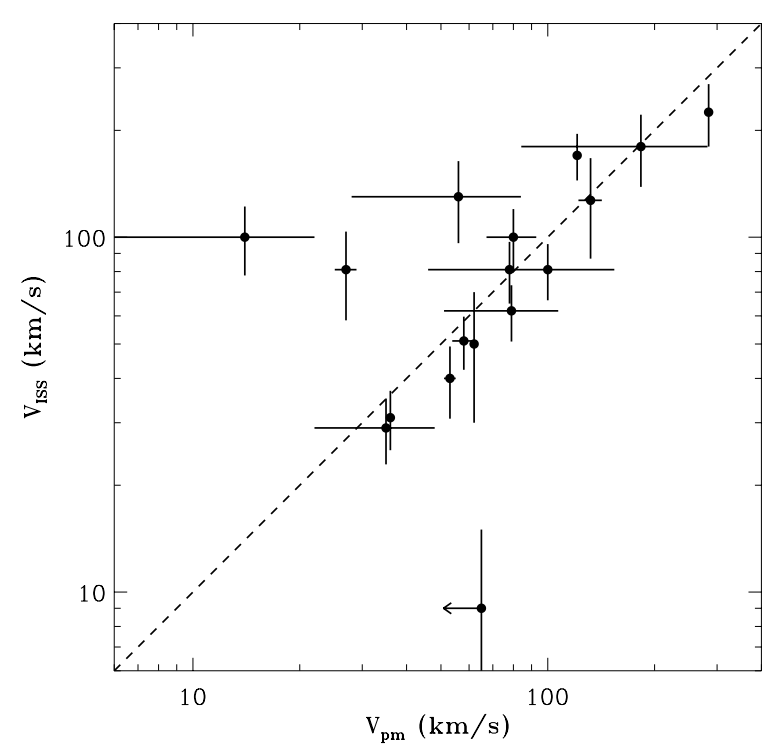

Fig. 4. Scatter diagram for proper motion and scintillation speeds for 16 millisecond pulsars. The $V_{\mathrm{pm}} \equiv V_{\text {iss }}$ correlation line is shown

the findings of Bhat et al. (1998) about the LISM, but seems to not be in agreement with their proposed model for the electron distribution;

- it is unlikely its $V_{\perp}$ exceeds $30 \mathrm{~km} \mathrm{~s}^{-1}$;

- as a consequence of this, the contribution of $V_{\perp}$ to the observed spin-down rate through the Shklovskii effect is negligible and suggests the derived parameters $B, \tau$ and $\dot{E}$ are not affected by spurious effects. In particular $\dot{E}=(2.7 \div 3.4) 10^{33} \mathrm{erg} \mathrm{s}^{-1}$ and the observed X-ray luminosity are fully compatible with an X-ray efficiency factor $\eta=110^{-3}$. However, the low observed $L_{x}$ suggests this pulsar is somehow in between the two proposed classes of X-ray emitting pulsars (thermal polar caps and magnetospheric emission). Only high quality $\mathrm{X}$-ray spectral data will allow discrimination between these two emission mechanisms. The new generation X-ray observatories XMM-Newton and Chandra can provide these data.

To obtain accurate estimates of the scintillation parameters, very long observation times and large bandwidths are needed. Because of its high sensitivity and site location in the North, the Indian Giant Metre Radio Telescope (GMRT) is the most appropriate instrument for PSR J0030+0451 scintillation studies. For minimizing systematical errors, the observations must preferably be carried out at epochs when the projected Earth transverse orbital speed is low. Within a few years we should also be able to have an accurate timing measurement of $V_{\mathrm{pm}}$, although our results already show that PSR J0030+0451 is one of the slowest pulsars (with known $V_{\perp}$ ).

The pulsar sample for which proper motion and scintillation speeds are available was revised with new values for ordinary pulsars and 17 (recycled) millisecond pulsars.
We performed several different correlation analyses on the resulting sample of 77 objects and found:

- assuming the very simple model with the scattering screen placed midway between the Earth and the pulsar, the overall $V_{\mathrm{pm}}-V_{\text {iss }}$ correlation is $r \simeq 80 \%$; this value is much higher than the one reported by Gupta (1995);

- MSPs show a good correlation $r_{\mathrm{s}} \simeq 65 \%$ that becomes $\simeq 88 \%$ if two anomolous PSRs (J1603-7202 and J2051-0827), for which local enhanced electron densities were reported, are excluded. This confirms that interstellar scintillation parameters can be used as a probe of enhanced electron densities along the line of sight to PSRs;

- we confirm that multi-epoch scintillation observations wash out systematics and give reliable estimates of the pulsar transverse velocity.

Acknowledgements. F. N. wishes to thank IRA-CNR for technical support. The Australia Telescope is funded by the Commonwealth of Australia for operation as a National Facility managed by the CSIRO.

\section{References}

Becker, W., \& Trümper, J. 1997, A\&A, 326, 682

Becker, W., Trümper, J., Lommen, A. N., \& Backer, D. C. 2000, ApJ, 545, 1015

Bhat, N. D. R., Gupta, Y., \& Rao, A. P. 1998, ApJ, 500, 262

Bhat, N. D. R., Rao, A. P., \& Gupta, Y. 1999, ApJS, 121, 483

Britton, M. C., Gwinn, C. R., \& Ojeda, M. J. 1998, ApJ, 501, L101

Camilo, F., Thorsett, S. E., \& Kulkarni, S. R. 1994, ApJ, 421, L15

Cordes, J. M., Weisberg, J. M., \& Boriakoff, V. 1985, ApJ, 288, 221

Cordes, J. M. 1986, ApJ, 311, 183

D'Amico, N. 2000, in Pulsar Astronomy - 2000 and Beyond, 202th ASP Conf. Ser., ed. M. Kramer, N. Wex, \& R. Wielebinski (San Francisco: ASP), 27

Fomalont, E. B., Goss, W. M., Manchester, R. N., \& Lyne, A. G. 1997, MNRAS, 286, 81

Fomalont, E. B., Goss, W. M., Beasley, A. J., \& Chatterjee, S. 1999, ApJ, 117, 3025

Gothoskar, P., \& Gupta, Y. 2000, ApJ, 531, 345

Gupta, Y., Rickett, B. J., \& Lyne, A. G. 1994, MNRAS, 269, 1035

Gupta, Y. 1995, ApJ, 451, 717

Harrison, P. A., \& Lyne, A. G. 1993, MNRAS, 265, 778

Johnston, S., Nicastro, L., \& Koribalski, B. 1998, MNRAS, 297, 108

Kawai, N., \& Saito, Y. 1999, Astro. Lett. Comm., 38, 1

Lange, Ch., Wex, N., Kramer, M., Doroshenko, O., \& Backer, D. C. 2000, in Pulsar Astronomy - 2000 and Beyond, 202th ASP Conf. Ser., ed. M. Kramer, N. Wex, \& R. Wielebinski (San Francisco: ASP), 61

Lommen, A. N., Zepka, A., Backer, D. C., et al. 2000, ApJ, 545, 1007 
Lumiella, V. 2000, Tesi di Laurea, Università degli Studi di Bologna, Italy

Lorimer, D. R., Lyne, A. G., Festin, L., \& Nicastro, L. 1995, Nature, 376, 393

McClure-Griffiths, N. M., Johnston, S., Stinebring, D. R., \& Nicastro, L. 1998, MNRAS, 492, L49

Nicastro, L., \& Johnston, S. 1995, MNRAS, 273, 122

Nigro, F. 2000, Tesi di Laurea, Università degli Studi di Bologna, Italy

Press, W., Teukolsky, S., Vetterling, W., \& Flannery, B. 1992, Numerical recipes in Fortran: The art of scientific computing, 2nd edition (Cambridge University Press), 633

Scheuer, P. A. G. 1968, Nature, 218, 920

Shklovskii, I. S. 1970, Soviet Astron., 13, 562
Somer, A. 2000, in Pulsar Astronomy - 2000 and Beyond, 202th ASP Conf. Ser., ed. M. Kramer, N. Wex, \& R. Wielebinski (San Francisco: ASP), 17

Stairs, I. H., Arzoumanian, Z., Camilo, F., et al. 1998, ApJ, 505,352

Stappers, B. W., Bailes, M., Lyne, A. G., et al. 1996, ApJ, 465, L119

Tauris, T. M., Savonije, G. J. 1999, A\&A, 350, 928

Taylor, J. H., \& Cordes, J. M. 1993, ApJ, 411, 674

Toscano, M., Britton, M. C., Manchester, R. N., Bailes, M., \& Sandhu, J. S. 1999a, ApJ, 523, L171

Toscano, M., Sandhu, J. S., Bailes, M., et al. 1999b, MNRAS, 307,925

van den Heuvel, E. P. J. 1994, A\&A, 291, L39 